Original Research Paper

\title{
Introducing a New Kit based on Modified Chromotropic Acid Method for Easy Determination of Methanol
}

\author{
${ }^{1}$ Mohammad Reza Ghadirzadeh, ${ }^{2}$ Ali Rafizadeh, ${ }^{1}$ Akbar Fattahi, \\ ${ }^{3}$ Seyed Davood Mirtorabi, ${ }^{4}$ Hajar Nazari and ${ }^{5}$ Melika Rafizadeh \\ ${ }^{1}$ Legal Medicine Research Center, Legal Medicine Organization, Tehran, Iran \\ ${ }^{2}$ Faculty of Nursing and Midwifery, Islamic Azad University, Rasht Branch, Rasht, Iran \\ ${ }^{3}$ Department of Neuroscience and Addiction Studies, School of Advanced Technologies in Medicine, \\ Tehran University of Medical Sciences, Tehran, Iran \\ ${ }^{4}$ Department of Epidemiology, Student Research Committee, Iran University of Medical Sciences, Tehran, Iran \\ ${ }^{5}$ Faculty of Pharmacy, Student Research Committee, Mazandran University of Medical Sciences, Ramsar, Iran
}

\section{Article history}

Received: 27-01-2019

Revised: $15-04-2019$

Accepted: 02-05-2019

Corresponding Author:

Ali Rafizadeh,

Islamic Azad University,

Rasht, Iran

Email: alirafizadeh@iaurasht.ac.ir

\begin{abstract}
Methyl alcohol poisoning usually happens by use of contaminated ethanol. So, development of an easy and cost-effective method to determine methanol in these products can be helpful to diagnosemethanolintoxication. The aim of this paper is introducing a new kit based on chromotropic acid method for measuring the methanol content of alcoholic beverages. In this study, a new modified chromotropic acid method (as a kit) was used to determine of methanol in 700 self-made samples with diverse ethanol concentration prepared by "add found" technique. Briefly, in the proposed method, produced formaldehyde by methanol oxidation is reacted with chromotropic acid in a high acidic media. The quantification limit of this kit lies below the permitted dose and safe amount of methanol in the beverages regulated by the European Parliament and the Council. The attained results indicate Limit of Quantification of the method is $1250 \mathrm{mg} / \mathrm{Land}$ all examined samples with more methanol amounts are easily determined with good accuracy and precision. As for the European standard about permitted dose of methanol in beverages (up to $4000 \mathrm{mg} /$ lin $40 \% \mathrm{v} / \mathrm{v}$ of alcohol strength) and gained results, it seems, this proposed method practically enables rapid and easy quantitative determination of methanol in all kind of alcoholic strength with suitable accuracy and precision. However, conclusive conclusions in this area will require further examination in actual samples of alcoholic beverages. But, to the best of our knowledge, there isn't any report about such easy method.
\end{abstract}

Keywords: Alcohol, Chromotropic Acid Method, Kit, Methanol, Poisoning

\section{Introduction}

Drinking of nonstandard alcoholic beverages contaminated with methanol may cause methanol intoxication (Shadnia et al., 2013). Methanol metabolites in human body can cause severe metabolic and neurological side effects that may lead to coma and even death (Rafizadeh et al., 2010; Brent, 2009; Paasma et al., 2012; Rostrup et al., 2016). Most alcoholic drinks contain methanol in low levels that does not cause side effects (Croitoru et al., 2013; Lachenmeier et al., 2006; Paine and Davan, 2001). In addition, there are regulations that control harmful contents of alcoholic drinks (Lachenmeier et al., 2011). Many authorities have set tolerable methanol limits in alcoholic drinks for example European Union Commission accepts up to $0.4 \%(\mathrm{~V} / \mathrm{V})$ methanol in an alcoholic drink with $40 \%$ ethanol (Croitoru et al., 2013; Paine and Davan, 2001). However, these limits and regulations are applied on recorded alcoholic manufactures and homemade drinks are not covered by these regulations (Rafizadeh et al., 2010; Lachenmeier et al., 2011).

Methanol toxicities may be a result of accidental ingestion of non-drinkable alcohols (Salek et al., 2014) or consumption of contaminated alcoholic preparations (Rostrup et al., 2016). In countries where alcohol drink and distribution are banned, smuggling of homemade and unsupervised alcoholic preparations is a profitable trade (Shadnia et al., 2013; Rafizadeh et al., 2010; Rostrup et al., 2016). Homemade Alcoholic beverages are sometimes 
intentionally contaminated with methanol for financial benefits (Rostrup et al., 2016) and cause methanol toxicity outbreaks (Hassanian-Moghaddam et al., 2015).

Chromotropic Acid (CA) colorimetric method for methanol detection in spirits has been recommended by Association of Official Analytical Chemists (AOAC) (Rafizadeh et al., 2010; Vaskova, 2014; HassanianMoghaddam et al., 2018). This reference method has some limitations. It is time consuming and needs large volume of hot concentrated sulfuric acid which is potentially hazardous and corrosive (Fagnani et al., 2003). Unfortunately, we face outbreaks of methanol toxicity due to smuggling of methanol contaminated alcoholic beverages in Iran (Hassanian-Moghaddam et $a l ., 2015)$ and existence of a reliable method for easy identification and quantification of methanol in such situations is a great advantage. In this paper a new selfmade kit based on modified CA method for easy determination of methanol in beverages is introduced which is much easier to use than the reference method. Precision and accuracy of this kit are determined by its comparison with pretreatment methanol amounts (add found method) (Alfassi, 1998; Dean, 1995) as gold standard. Also, the study of different concentrations of ethanol on possible results is another aim of our study.

\section{Materials and Methods}

In this study, methanol contents of 700 self-made alcoholic samples (resembling unrecorded beverages) prepared by add found technique in distillated water (D.W) were accidentally examined. The examiners of samples were blind about the real pre-determined concentration of methanol and finally, attained results by the kit were compared with initial contents of samples' methanol. Analysis was performed by Excel 2012 software.

\section{Instrumentation}

A single beam spectrophotometer-UV/VIS (Jenway 6405 , England) was used to determine methanol content in samples.

\section{Chemicals}

The needed methanol and ethanol for preparation of samples were purchased from Merck Company in Iran. Our newly designed specific kit produced by Arya Mabna Tashkhis Co., Tehran, Iran, was used to determine methanol content of the samples. This kit contains five reactants (A, B, C, D and E), five standards of methanol with concentrations of $0,12.5,25,50$, and $100 \mathrm{mg} / \mathrm{l}$, and an instruction brochure. Furthermore, a high quality of de-ionic distillated water (D.W) was used for preparation and dilution of samples.

\section{Preparation}

In this study, different amounts of ethanol were added to D.W to prepare samples with diverse concentrations $(20-72 \%)$ of it and then, they were again contaminated by methanol with concentrations of 1564.2-19552.5 mg/l. Finally, we had 70 groups of preparations with 10 in each group with clear methanol contents before using new kit. Three control solutions with 1250, 10000 and $20000 \mathrm{mg} /$ lof methanol in alcohol $40 \% \mathrm{v} / \mathrm{v}$ were also prepared with the same method and tested to define Limit of Quantification (LOQ) of the method. To perform the test, each sample was diluted (1:100 ratio) with D.W as triplicate and examined by proposed kit method.

\section{Procedure}

Based on the kit brochure, $50 \mu$ of each standard and all diluted (1:100) samples were poured into separated previously labeled test tubes with $50 \mu \mathrm{l} \mathrm{A}$ and $100 \mu \mathrm{l} \mathrm{B}$ reactants (sulfuric acid and potassium permanganate solutions) and shaken. Fifteen minutes later, $50 \mu \mathrm{l}$ of $\mathrm{C}$ reactant (sodium hydrogen sulfite solution) was added to the test tubes and they were shaken hardly to fade the color. Fifty $\mu \mathrm{l}$ of $\mathrm{D}$ reactant (CA solution) and $1 \mathrm{ml}$ of $\mathrm{E}$ reactant (concentrated sulfuric acid) were then added to the test tubes and they were shaken. After spontaneous cooling of test tubes at room temperature, the absorbance of each test tube was read at $575 \mathrm{~nm}$ and then, the methanol content of each sample was computed in comparison with the standard curve by multiplying the result by the dilution factor (100).

\section{Statistical Analysis}

At first, the means and standard deviations of kit results were compared with pre-determined values of methanol level (as gold standard). Then, the results were discussed as Relative Standard Deviation (RSD) and Relative Mean Error (RME) were calculated to determine quality assurance of the kit.

\section{Results}

The accuracy and precision were used to confirm the proposed kit efficacy (Bioanalytical Method Validation Guidance for Industry, 2017). The Table 1 shows the used kitanalytical quality assurance in ethanol-based solutions. As it is visiblein Fig. 1, the kit standard curve has good linearity with high coefficient of correlation (more than 0.99). Also, the kit has a good accuracy and precision to determine methanol content of the samples (Table 1).

To measure accuracy of the kit, test was applied on 700 self-made aqueous ethanol solutions. The methanol concentrations of preparations increased respectively from 1564.2 in the first group of samples to $19552.5 \mathrm{mg} /$ lin the 70th group. The ethanol concentrations of samples in each group (from 1th to 10th sample) varied from $20 \% \mathrm{~V} / \mathrm{V}$ to $72 \% \mathrm{~V} / \mathrm{V}$. All real and determined methanol concentrations (mean of methanol content of 10 samples in each group obtained by our new kit) are shown in Table 2. 
Mohammad Reza Ghadirzadeh et al. / American Journal of Pharmacology and Toxicology 2019, Volume 14: 1.6 DOI: 10.3844/ajptsp.2019.1.6

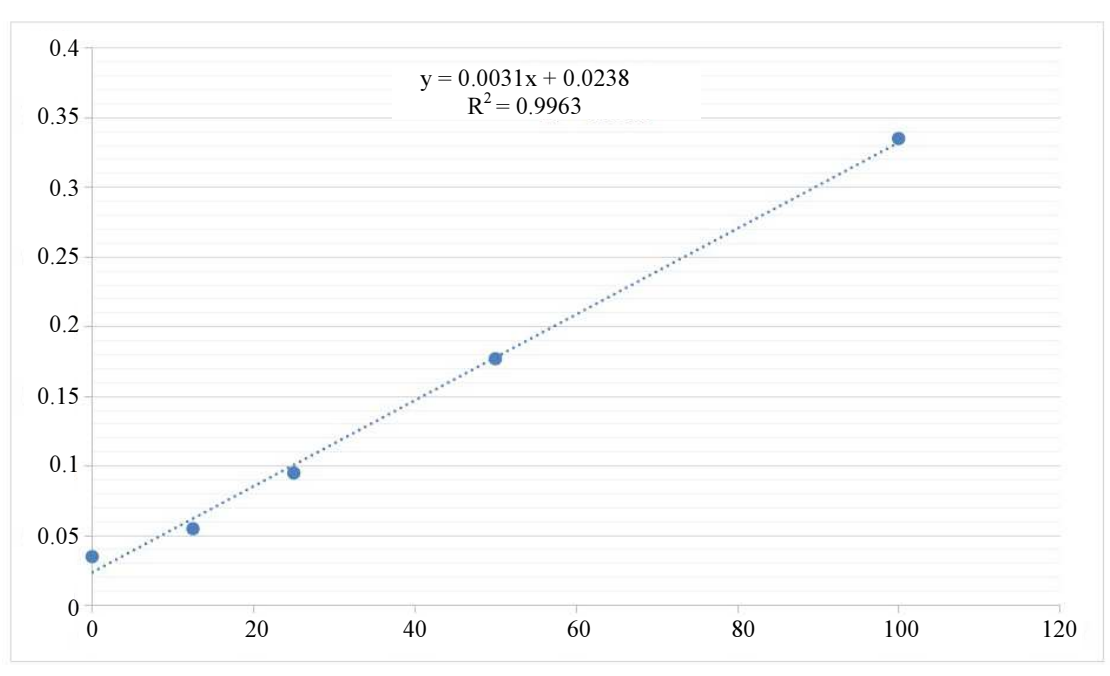

Fig. 1: Methanol calibration curve

Table 1: Precision of proposed method

\begin{tabular}{|c|c|c|c|c|}
\hline \multirow{2}{*}{$\begin{array}{l}\text { Methanol } \\
\text { Concentration }(\mathrm{mg} / \mathrm{L})\end{array}$} & \multicolumn{2}{|c|}{ Intraday $(n=5)$} & \multicolumn{2}{|c|}{ Inter-day $(n=5)$} \\
\hline & RME\% & $\mathrm{RSD} \%$ & RME\% & $\mathrm{RSD} \%$ \\
\hline 1250 & $3.1 \%$ & $2.4 \%$ & $3.4 \%$ & $4.9 \%$ \\
\hline 10000 & $0.6 \%$ & $0.8 \%$ & $1.9 \%$ & $2.6 \%$ \\
\hline 20000 & $0.3 \%$ & $0.5 \%$ & $1.1 \%$ & $2.9 \%$ \\
\hline
\end{tabular}

LOD: Limit of Detection $=700 \mathrm{mg} / \mathrm{l}$

LOQ: Limit of Quantification $=1250 \mathrm{mg} / 1$

Table 2: Results of analysis of methanol in 70 groups of samples with our kit

\begin{tabular}{|c|c|c|c|c|}
\hline \multirow[b]{2}{*}{ Group } & \multicolumn{2}{|c|}{ Methanol concentration } & \multirow[b]{2}{*}{ RME (\%) } & \multirow[b]{2}{*}{$\operatorname{RSD}(\%)$} \\
\hline & True values & New kit & & \\
\hline 1 & 1564.2 & 1338.1 & -14.5 & 4.7 \\
\hline 2 & 1759.7 & 1644.7 & -6.5 & 3.8 \\
\hline 3 & 1955.3 & 2003.7 & 2.5 & 3.2 \\
\hline 4 & 2737.4 & 3172.0 & 15.9 & 1.9 \\
\hline 5 & 3323.9 & 3774.8 & 13.6 & 4.5 \\
\hline 6 & 2541.8 & 3218.9 & 26.6 & 1.2 \\
\hline 7 & 2346.3 & 3173.0 & 35.2 & 3.7 \\
\hline 8 & 2150.8 & 2608.1 & 21.3 & 6.2 \\
\hline 9 & 3715.0 & 4370.9 & 17.7 & 5.3 \\
\hline 10 & 3519.5 & 4047.3 & 15.0 & 2.1 \\
\hline 11 & 3128.4 & 3828.1 & 22.4 & 3.2 \\
\hline 12 & 2932.9 & 3676.5 & 25.4 & 1.9 \\
\hline 13 & 5132.5 & 4885.0 & -4.8 & 3.2 \\
\hline 14 & 4154.9 & 4465.3 & 7.5 & 3.0 \\
\hline 15 & 4399.3 & 4578.5 & 4.1 & 2.9 \\
\hline 16 & 3910.5 & 4482.0 & 14.6 & 4.4 \\
\hline 17 & 7332.2 & 7102.5 & -3.1 & 2.1 \\
\hline 18 & 4643.7 & 4530.5 & -2.4 & 4.0 \\
\hline 19 & 4888.1 & 4657.1 & -4.7 & 2.1 \\
\hline 20 & 5621.3 & 4934.7 & -12.2 & 4.4 \\
\hline 21 & 5376.9 & 4766.0 & -11.4 & 3.3 \\
\hline 22 & 5865.8 & 5915.3 & 0.8 & 1.0 \\
\hline 23 & 6110.2 & 5396.8 & -11.7 & 1.7 \\
\hline 24 & 6354.6 & 5565.6 & -12.4 & 3.5 \\
\hline 25 & 6599.0 & 5876.9 & -10.9 & 1.4 \\
\hline
\end{tabular}




\begin{tabular}{|c|c|c|c|c|}
\hline 26 & 8798.6 & 8973.9 & 2.0 & 1.3 \\
\hline 27 & 7576.6 & 8027.4 & 5.9 & 2.0 \\
\hline 28 & 8065.4 & 8223.1 & 2.0 & 3.3 \\
\hline 29 & 7821.0 & 8148.1 & 4.2 & 3.8 \\
\hline 30 & 9043.0 & 9287.8 & 2.7 & 1.4 \\
\hline 31 & 9776.3 & 10020.5 & 2.5 & 1.6 \\
\hline 32 & 8309.8 & 8833.5 & 6.3 & 2.7 \\
\hline 33 & 8554.2 & 8870.8 & 3.7 & 0.7 \\
\hline 34 & 9287.4 & 9848.3 & 6.0 & 2.0 \\
\hline 35 & 10753.9 & 10356.8 & -3.7 & 1.1 \\
\hline 36 & 6843.4 & 7454.4 & 8.9 & 0.7 \\
\hline 37 & 11144.9 & 10509.3 & -5.7 & 1.3 \\
\hline 38 & 7087.8 & 6984.0 & -1.5 & 3.6 \\
\hline 39 & 9971.8 & 10033.3 & 0.6 & 2.4 \\
\hline 40 & 11927.0 & 11020.2 & -7.6 & 3.9 \\
\hline 41 & 10362.8 & 10296.7 & -0.6 & 0.7 \\
\hline 42 & 11536.0 & 10726.6 & -7.0 & 2.5 \\
\hline 43 & 13100.2 & 13504.8 & 3.1 & 1.2 \\
\hline 44 & 12318.1 & 12191.1 & -1.0 & 2.3 \\
\hline 45 & 15642.0 & 15713.0 & 0.5 & 1.1 \\
\hline 46 & 12709.1 & 12316.5 & -3.1 & 2.1 \\
\hline 47 & 14664.4 & 14890.0 & 1.5 & 0.7 \\
\hline 48 & 13491.2 & 13545.7 & 0.4 & 2.6 \\
\hline 49 & 15837.5 & 15799.8 & -0.2 & 0.8 \\
\hline 50 & 13882.3 & 14216.5 & 2.4 & 0.6 \\
\hline 51 & 16815.2 & 16067.8 & -4.4 & 4.0 \\
\hline 52 & 16424.1 & 16033.2 & -2.4 & 2.3 \\
\hline 53 & 14468.9 & 14858.4 & 2.7 & 1.6 \\
\hline 54 & 14859.9 & 15033.2 & 1.2 & 3.1 \\
\hline 55 & 14273.3 & 14635.8 & 2.5 & 0.4 \\
\hline 56 & 15055.4 & 15224.8 & 1.1 & 4.2 \\
\hline 57 & 15446.5 & 15477.4 & 0.2 & 0.9 \\
\hline 58 & 16228.6 & 15870.7 & -2.2 & 3.5 \\
\hline 59 & 16619.6 & 16390.9 & -1.4 & 1.0 \\
\hline 60 & 15251.0 & 15380.0 & 0.8 & 2.4 \\
\hline 61 & 17010.7 & 16539.0 & -2.8 & 0.6 \\
\hline 62 & 16033.1 & 15800.0 & -1.5 & 1.5 \\
\hline 63 & 17597.3 & 18295.6 & 4.0 & 1.2 \\
\hline 64 & 17206.2 & 16643.4 & -3.3 & 0.5 \\
\hline 65 & 18086.1 & 18444.5 & 2.0 & 0.9 \\
\hline 66 & 17401.7 & 16993.4 & -2.3 & 1.4 \\
\hline 67 & 18574.9 & 18613.9 & 0.2 & 0.7 \\
\hline 68 & 17792.8 & 19090.0 & 7.3 & 1.2 \\
\hline 69 & 19063.7 & 18159.4 & -4.7 & 0.6 \\
\hline 70 & 19552.5 & 22593.4 & 15.6 & 0.9 \\
\hline
\end{tabular}

RME: Relative Mean Error

RSD: Relative Standard Deviation

Notice: all of concentrations are based on $\mathrm{mg} / \mathrm{l}$

The comparison of methanol concentrations obtained by new kit with previous definite methanol levels (gold standard) (Alfassi, 1998; Dean, 1995) shows the acceptability of the new method. Because, the means of real and determined methanol levels in 70 series of examined samples were similar (10060 and $10144 \mathrm{mg} / \mathrm{l}$ respectively). Mean of RSDs of examined groups was
$2.3 \%$ that is indicative of high precision. This calculation is reinforced by observation of just two (2.86\%) RSDs more than $5 \%$ in 70 groups of examined samples which demonstrates $97.14 \%$ of examined samples have acceptable RSD and henceit is deductible, the purposed kit has high precision. Also, mean of RMEs of examined groups was $6.6 \%$ and 27 (38.6\%) REs were more than 5\%. 
Although this finding requires more investigation, it shows, $61.4 \%$ of examined groups have acceptable RE and hence it seems, the kit has a relatively suitable accuracy.

\section{Discussion}

High levels of methanol in alcoholic drinks can lead to methanol toxicity that is accompanied by severe symptoms, different organ failures (especially, blindness) and death. Determination of methanol content in alcoholic products is important in quality control of formal alcoholic beverages. In contrast, homemade or traditionally produced alcoholic drinks are not monitored for methanol. Common methods used for determination of methanol in alcoholic drinks such as High Performance Liquid Chromatography (HPLC), gas Chromatography (GC) and GC-MS (usually GC) are not easily applicable in developing countries with limited financial and expert resources (Hassanian-Moghaddam et al., 2018). Introduction and use of a feasible method for quantification of methanol in developing countries is an advantage. We could not find many report similar to our study but Hassanian-Moghaddam et al. (2018) were evaluated the same kit in real samples (alcoholic beverages) (Hassanian-Moghaddam et al., 2018). Unfortunately, we were not able to compare the present results with more previous studies.

Methanol concentrations of all self-made samples determined by our new kit were close to their real levels and our method had acceptable precision in every concentration of ethanol. Also, the gained results were shown, the sensitivity of proposed method is not affected by ethanol concentration and methanol content is independently determined in aqueous media containing different concentrations of ethanol. So, from this point of view, the proposed kit has enough credit and can be applied in similar cases. However, the accuracy of the kit was not ideal (total $\mathrm{RME}=6.6 \%$ ) that can be due to some errors and needs more investigation.

In reference colorimetric CA method which is recommended as the standard method for determination of methanol in alcoholic drinks by AOAC (Rafizadeh et al., 2010; Vaskova, 2014; Hassanian-Moghaddam et al., 2018), methanol is changed to formaldehyde (HCHO) and this compound determined indirectly by its reaction with $\mathrm{CA}$ in hot concentrated sulfuric acid media (Fagnani et al., 2003; Mohammed et al., 2008). Thus, the formaldehyde and formic acid contents of the sample always influence the amount of determined methanol level if they simultaneously exist in the sample. Therefore, to obtain higher levels of methanol in real samples (alcoholic beverages) by this kit than the other current methods are possible, however, confirmation of it needs more investigation. Perhaps, this feature reduces the specificity of the CA method, but given the fact that formaldehyde and formic acid are the main toxic metabolites of methanol in human body and cause methanol poisoning symptoms, it looks like an advantage. On the other hand, certainly, AOAC with full knowledge of mentioned points has recommended this method as a reference technique for determination of methanol in alcoholic beverages (16).

All above mentioned points demonstrate that the proposed method (kit) in this study has enough credit and ability to determine methanol in alcoholic beverages with different concentrations of ethanol and can be used as a trustable alternative tool instead of the other current for routine determination of methanol in these products by different users with limited facilities and minimum laboratory equipment. However, it should be to mention that we did not check the methanol content of the samples by advanced methods (like GC) and this is probably the major limitation of the current study. Also, useless of real alcoholic beverages as sample was the second major limitation of our study that must be done in future. But, introducing a non-common reference method (add found technique) for evaluation of a new chemical method seems to be one of the most important feature of this study.

\section{Conclusion}

Our study was shown; the new used kit has acceptable sensitivity and accuracy for easy quantification of methanol in alcoholic drinks with no need to advanced laboratory equipment, professional knowledge and financial resources. However, the final judgment about its feasibility needs more investigations on real samples in comparison with other gold standards like GC.

\section{Acknowledgement}

The authors would like to thank Arya Mabna Tashkhis Co., Tehran, Iran, for financial and technical supports.

\section{Funding Information}

This study was funded by the Iranian Legal Medicine Organization.

\section{Author's Contributions}

Mohammad Reza Ghadirzadeh: Contributed in writing proposals, final report and also, representation of scientific comments.

Ali Rafizadeh: Contributed in the preparation of proposals, final report and also, performance of experiments.

Akbar Fattahi: Contributed in pretreatment and making of self-made samples.

Seyed Davood Mirtorabi: Contributed in writing proposals, final report and also, representation of scientific comments. 
Hajar Nazari: Contributed in performance of statistical analysis of data.

Melika Rafizadeh: Contributed in preparing of samples and controls to performance of tests.

\section{Ethics}

This article does not contain any studies with human participants or animals performed by any of the authors.

\section{References}

Alfassi, Z.B., 1998. Instrumental multi-element chemical analysis. Kluwer Academic Publishers. Bioanalytical Method Validation Guidance for Industry.

Brent, J., 2009. Fomepizole for ethylene glycol and methanol poisoning. N Engl. J. Med.

Croitoru, M.D., F.I. Topor Elena and E. Fogarasi, 2013. A survey on the methanol content of home distilled alcoholic beverages in Transylvania (Romania). Acta Medica Marisiensis.

Dean, J.A., 1995. Dean's Analytical Chemistry Handbook. 2nd Edn., McGraw-Hill handbooks.

Fagnani, E., C.Melios, L. Pezza and H.R. Pezza, 2003. Chromotropic acid-formaldehyde reaction in strongly acidic media. The Role of Dissolved Oxygen and Replacement of Concentrated Sulphuric Acid. Talanta.

Hassanian-Moghaddam, H., A. Nikfarjam, A. Mirafzal, A. Saberinia and A.A. Nasehi et al., 2015. Methanol mass poisoning in Iran: Role of case finding in outbreak management. J. Public Health.

Hassanian-Moghaddam, H., A. Rafizadeh, S. Shariati, M. Rafizadeh and N. Zamani, 2018. Evaluation of methanol content of beverages using an easy modified chromotropic acid method. Food Chem. Toxicol.

Lachenmeier, D.W., E.M. Sohnius, R. Attig and M.G. López, 2006. Quantification of selected volatile constituents and anions in Mexican Agave spirits (Tequila, Mezcal, Sotol, Bacanora). J. Agric. Food Chem.
Lachenmeier, D.W., K. Schoeberl, F. Kanteres, T. Kuballa and E.M. Sohnius et al., 2011. Is contaminated unrecorded alcohol a health problem in the European Union? A review of existing and methodological outline for future studies. Addiction.

Mohammed, A.A., A.T. Mubarak, Z.M.H. Marestani and K.F. Fawy, 2008. Highly sensitive and selective catalytic determination of formaldehyde and acetaldehyde. Talanta.

Paasma, R., K.E. Hovda, H. Hassanian-Moghaddam, N. Brahmi and R. Afshari et al., 2012. Risk factors related to poor outcome after methanol poisoning and the relation between outcome and antidotes-a multicenter study. Clin Toxicol (Phila).

Paine, A. and A.D. Davan, 2001. Defining a tolerable concentration of methanol in alcoholic drinks. Hum. Exp. Toxicol.

Rafizadeh, A., S. Shariati, L. Pourmohammad and S. Fooladmehr, 2010. Application a colorimetric method for qualitative analysis of methanol. Sci. J. Forensic Med.

Rostrup, M., J.K. Edwards, M. Abukalish, M. Ezzabi and D. Some et al., 2016. The methanol poisoning outbreaks in Libya 2013 and Kenya 2014. PloS one.

Salek, T., P. Humpolicek and P. Ponizil, 2014. Metabolic disorders due to methanol poisoning. Biomedical Papers.

Shadnia, S., M. Rahimi, K. Soltaninejad and A. Nilli, 2013. Role of clinical and paraclinical manifestations of methanol poisoning in outcome prediction. J. Res. Med. Sci.

Vaskova, H., 2014. Spectroscopic determination of methanol content in alcoholic drinks. Int. J. Biology Biomedical. Eng. 